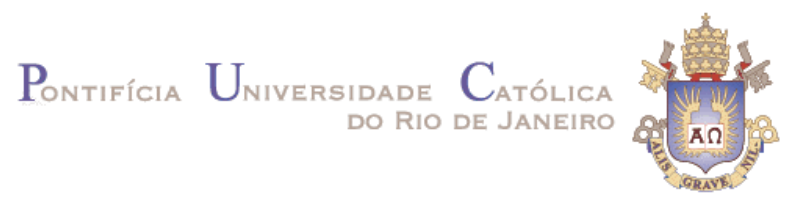

Gustavo Bahury Mesquita

\title{
Gestão de Capital de Giro: Uma Aplicação do Modelo Fleuriet a Empresas Argentinas, Brasileiras, Chilenas e Mexicanas
}

\section{Dissertação de Mestrado}

Dissertação apresentada ao Programa de PósGraduação em Administração de Empresas da PUC-Rio como requisito parcial para obtenção do título de Mestre em Administração de Empresas. .

Orientador: Prof. Roberto Moreno Moreira 


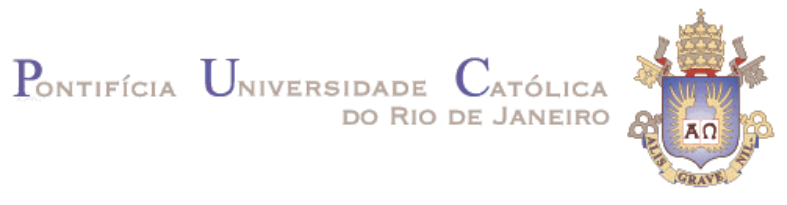

Gustavo Bahury Mesquita

Gestão de Capital de Giro: Uma Aplicação do Modelo Fleuriet a Empresas Argentinas, Brasileiras, Chilenas e Mexicanas

Dissertação apresentada como requisito parcial para obtenção do grau de Mestre pelo Programa de PósGraduação em Administração de Empresas da PUCRio. Aprovada pela Comissão Examinadora abaixo assinada.

\author{
Prof. Roberto Moreno Moreira \\ Orientador \\ Departamento de Administração de Empresas - PUC-Rio \\ Prof. Marcelo Cabus Klozle \\ Departamento de Administração de Empresas - PUC-Rio \\ Prof. Antonio de Araujo Freitas Junior \\ FGV \\ Prof. Nizar Messari \\ Coordenador(a) Setorial do Centro \\ de Ciências Sociais - PUC-Rio
}


Todos os direitos reservados. É proibida a reprodução total ou parcial do trabalho sem autorização da universidade, do autor e do orientador.

\section{Gustavo Bahury Mesquita}

Graduou-se em Administração de Empresas na PUC-Rio em 2001. Trabalhou na área financeira da IBM Brasil de 1999 até o primeiro semestre de 2008, atualmente trabalha na área financeira da IBM Corporação em Nova lorque, EUA.

\section{Mesquita, Gustavo Bahury}

Gestão de capital de giro : uma aplicação do modelo Fleuriet a empresas argentinas, brasileiras, chilenas e mexicanas / Gustavo Bahury Mesquita ; orientador: Roberto Moreno Moreira. - 2008.

239 f. : il. ; $30 \mathrm{~cm}$

Dissertação (Mestrado em Administração)Pontifícia Universidade Católica do Rio de Janeiro, Rio de Janeiro, 2008.

Inclui bibliografia 
A Deus e meus amigos e protetores espirituais e a minha família por todo o apoio, amor e carinho. 


\section{Agradecimentos}

Assim como a dedicatória os agradecimentos devem ser a Deus, meus amigos e protetores espirituais e a minha família por todo o apoio, amor e carinho.

Ao Professor Moreno por toda a sua ajuda, boa vontade e compreensão durante esse caminho. 


\section{Resumo}

Mesquita, Gustavo Bahury; Moreira, Roberto Moreno. Gestão de Capital de Giro: Uma Aplicação do Modelo Fleuriet a Empresas Argentinas, Brasileiras, Chilenas e Mexicanas. Rio de Janeiro, 2008. 239p. Dissertação de Mestrado - Departamento de Administração, Pontifícia Universidade Católica do Rio de Janeiro.

Nos níveis atuais de globalização, fluxo de informações e sensibilidade das economias e empresas em todo o mundo, e onde os mercados financeiros, os processos, operações e dinâmicas organizacionais são cada vez mais padronizados, torna-se cada vez mais importante a precisão dos indicadores e dados que são utilizados na avaliação de empresas para a percepção quanto as suas situações financeiras por meio da observação de indicadores de capital de giro, em especial, em novos mercados e países e para as empresas globalizadas. Este trabalho propõe um modelo de avaliação de situação econômico-financeiro de empresas baseado em uma analise conjunta da aplicação dos clássicos e tradicionais Indicadores de Liquidez com o Modelo Dinâmico de Capital de Giro, estudado e desenvolvido por Michel Fleruiet. O trabalho propõe sua aplicação em empresas de quatro países da America Latina, Argentina, Brasil, Chile e México. Testes aplicados sobre as Demonstrações Financeiras das empresas dos setores de tecnologia e energia representando cem por cento das empresas de capital aberto destes setores nos países revelaram que e benéfica a utilização conjunta dos modelos de avaliação de situação econômico-financeiro e de autofinanciamento.

\section{Palavras-chave}

Capital de Giro, America Latina, Modelo Fleuriet e Indicadores de Liquidez. 


\section{ABSTRACT}

Mesquita, Gustavo Bahury; Moreira, Roberto Moreno. Working Capital Management: An Aplication of the Fleuriet Model on Argentine, Brazilian, Chilean and Mexican Companies. Rio de Janeiro, 2008. 239p. MSc Dissertation - Departamento de Administração, Pontifícia Universidade Católica do Rio de Janeiro.

On the current globalization levels, information flow and economies and companies sensibility all over the world, and where financial markets, the procedures, operatives and organizational dynamics are at each more standardized, becomes as more important the indicators precision and the data that are used evaluating companies to perceive regarding its financial situations by the observation of the working capital indicators, specially over new markets and countries and for globalized companies. This study intend to propose an evaluation model for financial-economic companies situation based on a jointly application of the classics and traditional Liquid Indicators with the Dynamic Working Capital Model studied and developed by Michel Fleuriet. The studies propose to apply it over four Latin America countries, Argentina, Brazil, Chile and Mexico. Such trials accomplished over Financial Statements of technology and energy companies representing one hundred percent of open market capital companies of these segments on countries, revealed that it's beneficial the use of both models of evaluation of the financial-economic situation and auto-sufficiency jointly.

\section{Keywords}

Working Capital, Latin America, Fleuriet and Liquidity Indicators Models. 


\section{Sumário}

1 Introdução 13

1.1. Objetivos e Organização da Dissertação 15

2 Modelo de Liquidez $\quad 18$

2.1. Referencial Histórico sobre o Modelo Tradicional de Liquidez 18

$\begin{array}{ll}\text { 2.1.1. Índices de Liquidez } & 19\end{array}$

3 Modelo Dinâmico de Capital de Giro 24

3.1. Referencial Histórico do Modelo Dinamico de Capital de Giro 24

3.2. O Balanço Patrimonial, sua visão estática e dinâmica 26

3.3. Necessidade de Capital de Giro (NCG), Capital de Giro (CDG) e Saldos de Tesouraria (T) 30

3.4. Correlação das Variáveis, Autofinanciamento e Efeito Tesoura 34

3.4.1. Autofinanciamento 34

3.4.2. Efeito Tesoura 37

3.5. Determinação das Contas do Balanço em Erráticas, Cíclicas e Não Cíclicas $\quad 39$

3.6. Determinação e Avaliação das Estruturas Financeiras pela Combinação das Variáveis: NCG, CDG e T 43

3.7. Enquadramento por Situação e Tipo 47

4 Metodologia $\quad 49$

4.1. Amostra e Obtenção de Dados 49

4.2. Dados Expurgados da Analise $\quad 51$

5 Aplicação dos Modelos e Resultados 52

5.1. Empresas Argentinas 53

5.1.1. Indicadores das Empresas Argentinas 54

5.2. Empresas Brasileiras 56

5.2.1. Indicadores das Empresas Brasileiras 60

5.3. Empresas Chilenas $\quad 67$

5.3.1. Indicadores das Empresas Chilenas 68 
5.4. Empresas Mexicanas $\quad 72$

5.4.1. Indicadores das Empresas Mexicanas $\quad 72$

5.5. Avaliação dos Resultados 74

5.5.1. Quando a Liquidez 74

5.5.2. Quando ao Posicionamento no Modelo Dinâmico 75

5.6. Comparando e Conectando os Resultados e Classificações dos Modelos $\quad 76$

6 Conclusão $\quad 82$

7 Referencias Bibliograficas $\quad 85$

8 Anexo A - Demonstrações Financeiras das Empresas Argentinas 87

9 Anexo B - Demonstrações Financeiras das Empresas Brasileiras 109

10 Anexo C - Demonstrações Financeiras das Empresas Chilenas 188

11 Anexo D - Demonstrações Financeiras das Empresas Mexicanas 226 


\section{Lista de quadros}

Quadro 1-Demonstração dos Índices de Liquidez 21

Quadro 2-Estrutura Tradicional do Balanço 27

Quadro 3-Os Ciclos no Balanço Patrimonial 29

Quadro 4-Reorganização do Balanço Patrimonial 33

Quadro 5-A Dinâmica do Resultado Econômico 36

Quadro 6-Composição Patrimonial das Empresas TIPO 1

Quadro 7-Composição Patrimonial das Empresas TIPO 244

Quadro 8-Composição Patrimonial das Empresas TIPO $3 \quad 45$

Quadro 9-Composição Patrimonial das Empresas TIPO $4 \quad 46$

Quadro 10-Composição Patrimonial das Empresas TOPO $5 \quad 46$

Quadro 11-Composição Patrimonial das Empresas TIPO 6

Quadro 12-Posicionamento financeiro da empresa com base nas variáveis NCG, CDG e ST 48

Quadro 13-Fatores determinantes e influenciadores na alteração das variáveis do modelo dinâmico 48

Quadro 14-Relação das empresas Argentinas analisadas 53

Quadro 15-Indicadores de Liquidez e do Modelo Dinâmico nas Empresas Argentinas $\quad 55$

Quadro 16-Relação das Empresas Brasileiras 57

Quadro 17-Indicadores de Liquidez e do Modelo Dinâmico das $\begin{array}{ll}\text { Empresas Brasileiras } & 61\end{array}$

Quadro 18-Relação das Empresas Chilenas Analisadas 67

Quadro 19-Indicadores de Liquidez e do Modelo Dinâmico das Empresas Chilenas 69

Quadro 20-Relação das Empresas Mexicanas Analisadas 72

Quadro 21-Indicadores de Liquidez e do Modelo Dinâmico das $\begin{array}{ll}\text { Empresas Mexicanas } & 73\end{array}$

Quadro 22-Resultados Comparativos para Empresas Argentinas 78

Quadro 23-Resultados Comparativos para Empresas Brasileiras 79 
Quadro 24-Resultados Comparativos para Empresas Chilenas 


\section{Lista de Siglas}

AC-Ativos Circulantes

ACF-Ativos Circulantes Financeiros

ACO-Ativos Circulantes Operacionais

BRIC-Brasil, Russia, India e China

CCL-Capital Circulante Liquido

CDB-Certificado de Deposito Bancario

CDG-Capital de Giro

CESA-Centre d'Enseignement Superieur des Affaires

D-Disponibilidade

DI-Deposito Interfinanceiro

E-Estoques

EBITDA-Earnings Before Interest, Taxes, Depreciation and Amortization

ELP-Exigivel em Longo Prazo

IAG-Instituto de Administacao e Gerencia

LAJIRDA-Lucro Antes dos Juros, Imposto de Renda, Depreciacao e

Amortizacao

LBO-Lucro Bruto Operacional

NCG-Necessidade de Capital de Giro

PC-Passivo Circulante

PCF-Passivos Circulantes Financeiros

PCO-Passivos Circulantes Operacionais

PUC-Pontificia Universidade Catolica

RLP-Realizavel em Longo Prazo

ST-Saldos de Tesouraria

T-Tesouraria 